\title{
PROCESSES CONTROLLING THE INITIAL MASS FUNCTION: INTERSTELLAR TURBULENCE AND MAGNETIC FIELDS
}

\author{
R. C. FLECK, JR. \\ Embry-Riddle Aeronautical University \\ Daytona Beach, FL USA
}

\begin{abstract}
The observed flattening of the initial stellar mass function at low mass can be accounted for in terms of the different interstellar cloud size-mass scaling and different ambipolar diffusion time scaling for small, thermally-supported clouds and larger clouds supported primarily by turbulent pressure.
\end{abstract}

Following Reddish and Sloan (1971; see also Reddish 1978), we expect the mass spectrum for stars, $N_{*}(M)$, and for dense, interstellar cloud cores, $N_{c}(M)$, to be related by the rate at which these clouds form stars, $\mathrm{t}^{-1}$ :

$$
N_{*}(M) \propto N_{c}(M) t^{-1},
$$

where the cloud mass spectrum (cf. Scalo 1985; Benson and Myers 1989)

$$
\mathrm{N}_{\mathrm{c}}(\mathrm{M}) \propto \mathrm{M}^{-1.5 \pm 0.5}
$$

and the cloud-to-star conversion rate $\mathrm{t}^{-1}$, assumed by Reddish and Sloan to be the gravitational free-fall time, is here taken to be equal to the ambipolar diffusion time, inasmuch as interstellar clouds are now known to be both turbulent and magnetic (cf. Fleck 1988; Myers and Goodman 1988).

A recent, comprehensive model of ambipolar diffusion in turbulent magnetic clouds predicts that $\mathrm{t} \alpha \mathrm{R}^{0.5} \alpha \mathrm{M}^{0.25}$ for larger clouds (radius $\mathrm{R} \gtrsim 0.1 \mathrm{pc} ; \mathrm{M} \gtrsim$ few $M_{\odot}$ ), while $t \alpha R^{-1} \alpha M^{-1}$ for smaller clouds which are supported against gravity primarily by thermal gas pressure (Myers and Goodman 1988). Thus, the predicted stellar initial mass function (IMF), counting stars in logarithmic mass intervals, is

$$
\begin{aligned}
& N_{*}(\log M) \alpha N_{*}(M) M \quad \alpha M^{0.5} \pm 0.5 \text { for } M \leqq M_{\odot} \\
& \alpha M^{-0.75} \pm 0.5 \text { for } M \gtrsim \text { few } M_{\odot} \text {. }
\end{aligned}
$$


The agreement shown here in the accompanying diagram (subject to arbitrary normalization) with the field star IMF (Scalo 1986) is certainly suggestive and is, it appears, the first attempt to discern quantitatively the possible role of magnetic fields in determining the IMF. The flattening/ turnover at low mass can be accounted for in terms of the different size-mass scaling and ambipolar diffusion time scaling for small, thermally-supported clouds and large clouds supported primarily by "turbule.nt" pressure. This transition is characterized by a minimum in the diffusion time at a fragment mass of order $1 \mathbf{M}_{\odot}$, which translates to a maximum, cloud-to-star conversion rate at the transition mass and a concomitant tendency for the IMF to "peak" (i.e., turnover) in that vicinity. The influence of this "sonic" transition on the IMF turnover has been discussed previously by Fleck (1982).

While other processes such as mass accretion (loss) during the protostellar stage, which would flatten (steepen) the IMF, may also operate to "fine tune" the predictions here for the IMF, the evidence presented suggests that mechanisms associated with interstellar turbulence and magnetic fields may in fact be controlling factors for star formation in the Galaxy.

\section{REFERENCES}

Benson, P. J., and Myers, P. C. (1989) Ap. J. Suppl., 71, 89.

Fleck, R. C. (1982) M.N.R.A.S., 201, 551. . (1988) Ap. J., 328, 299.

Myers, P. C., and Goodman, A. A. (1988) Ap. J., 329, 392.

Reddish, V. C. (1978) Stellar Formation (Pergamon, Oxford).

Reddish, V. C., and Sloan, C. (1971) Observatory, 91, 70.

Scalo, J. M. (1985) in Protostars \& Planets II, eds. D. C. Black and M. S. Mathews (University of Arizona Press, Tucson), p. 201. . (1986) Fund. Cosmic Phys., 11, 1. 